Scientific Visualization, 2021, volume 13, number 2, pages 79 - 93, DOI: 10.26583/sv.13.2.06

\title{
Creative Map Studio: A Platform for Visual Analytics of Mental Maps
}

\author{
R.V. Chumakov', K.V. Ryabinin², K.I. Belousov3, J. Duan 4 \\ Perm State University, Perm, Russia \\ 1 ORCID: 0000-0002-6860-1932, chumakoff.r.v@gmail.com \\ 2 ORCID: oooo-0002-8353-7641, kostya.ryabinin@gmail.com \\ 3 ORCID: 0000-0003-4447-1288, belousovki@gmail.com \\ 4 ORCID: 0000-0003-3891-2016,707695802@qq.com
}

\begin{abstract}
This paper is devoted to the development of software tools, which allow to create and analyze mental maps in digital format. Mental maps are used to study humans' cognitive characteristics and geospatial perception. To support Digital Humanities research based on the analysis of the mental maps, we developed a special high-level web-based vector graphics editor called Creative Maps Studio that allows informants to intuitively draw digital naive maps as a representation of their mental maps.

The Creative Maps Studio web application provides a wide variety of drawing tools based on points, lines, and areas, which allow representing different objects on the map, such as cities, historical monuments, industrial facilities, state borders, roads, rivers, steppes, seas, swamps, etc. For each object, individual parameters can be set, such as color, size, transparency, name, description, emoticons, etc.

Digital maps can be analyzed in various ways, relying on computer vision, machine learning, visualization, and manual expert analysis. Creative Maps Studio stores not only the final state of maps but also the entire history of user actions on the canvas, which makes it possible to consider the process of creating a map by the analysis.

While Creative Maps Studio provides basic functions to view the informants' maps and the history of their creation, we propose involving special software to achieve a comprehensive analysis of mental maps. In particular, we rely on the previously developed SciVi data mining platform and Semograph graph-semantic modeling information system. Creative Maps Studio provides appropriate export capabilities to transfer required data to these software systems. After exporting, the expert can use SciVi and Semograph to perform visual analytics of map objects' properties, to handle maps' textual layers, and to classify the maps on the principles of the fuzzy set theory. Thus, the mentioned software can be used to carry out the comprehensive study of informants' spatial perception.

In the future works, we plan to expand the set of objects available to draw the maps, allowing researchers to create custom ones, as well as to integrate analytics tools of maps creation history directly in the Creative Maps Studio.
\end{abstract}

Keywords: Mental Maps, Naive Geography, Fuzzy Classification, Pattern Matching, Circular Graph, Visual Analytics, Digital Humanities. 


\section{Introduction}

Geographic mental maps as ways and forms of organizing mental representations of space $[1,2]$ are of interest for a wide range of scientific disciplines since they reveal the humans' receiving, processing, storing, and broadcasting of individual ideas about the world. From a social point of view, geo-mental maps provide models for understanding/mastering the spatial macroenvironment and the behavior of various social groups in that environment, while the groups are distinguished by gender, age, nationality, residence region, etc. [3-5].

To efficiently analyze the mental maps, an adequate representation form should be chosen to convey the composition, metrics, and connections of objects contained in mental maps. In this regard, the most promising way is the visualization of informants' subjective geospatial representations. This visualization can be carried out in the paradigm of "naive" geography: in the form of the so-called "naive" maps (another term "sketch maps" is also common $[6,7])$. M. Blades has shown that naive maps have the feature of sustainable reproduction [8], which means that the same informant draws nearly the same naive map time after time. This indicates, naive maps are an adequate reflection of mental maps, therefore they can be treated as an objective measure of an informant's attitude to his/her spatial surrounding. As a result, naive maps are valuable material for the Digital Humanities research, allowing to monitor and justify the public moods in a particular region or social group $[1,3]$, as well as to study individual cognitive features of informants and the way they perceive the world around them.

Traditionally, the naive maps are drawn on paper. This is quite convenient for the informants, but it has two significant drawbacks. Firstly, in order to benefit the modern computational processing methods, the paper-drawn maps should be completely digitized (including the annotating of all available objects on each map). Secondly, it is required to normalize the drawing process, including the use of standardized technical and symbolic means. The variety of representation forms of naive maps can be clearly seen in the gallery of regional maps of Russia: https://naivemaps.ru/?page $\mathrm{id}=395$. It is a challenging task to handle these images, while fully automatic processing and interpretation for a sake of data mining are nearly impossible. Moreover, paper-drawn naive maps do not contain information about the process of their creation, including the sequence of adding objects and their characteristics.

To overcome the shortcomings mentioned above, we developed the Creative Maps Studio web application (https://creativemaps.studio/). This application is a web-based vector graphics editor, in which informants visualize their spatial representations in a form of digital naive maps using the predefined set of drawing tools. For the experts in Digital Humanities, the Creative Maps Studio provides functions to export naive maps composed by the informants for subsequent data mining and visual analytics. The analytics is performed outside of Creative Maps Studio, in the SciVi data mining platform and Semograph graph-semantic modeling information system. These two software systems were developed earlier and proved to be efficient and flexible in analyzing complex data [9]. Creative Maps Studio can be used by researchers from various fields of science, such as sociology, politology, tourismrelated sciences, etc. 


\section{Key Contributions}

In this paper, we propose a special vector graphics editor to create digital naive maps, as well as the following ways to analyze these maps within a remit of Digital Humanities research:

1. Analysis of the naive map creation history using Creative Maps Studio. All the manipulations with maps' objects in chronological order can be viewed, which enables studying the process of building the map, including the tracking of objects, which were created, but afterward deleted and thereby not presented in the final map state.

2. Visual analysis of naive maps in SciVi data mining platform using a circular graph that allows revealing relationships between map objects, as well as correlations between maps' structure and socio-cognitive parameters of the informants.

3. Semantic analysis of naive maps using the Semograph graph-semantic modeling information system. This opens up the possibility of automated analyzing names, descriptions, and comments of map objects.

4. Fuzzy classification of naive maps based on weighted fuzzy pattern matching. This method makes it possible to quantify the features of the spatial perception of informants from different regions and to test the similarity of the given map with the typical maps of a certain region.

\section{Related Work}

The first studies of mental maps emerged in the second half of the 2oth century and were carried out using naive maps $[10,11]$. For a long time, research was carried out without the use of computer technology. Naive maps drawn on paper were used and, accordingly, the analysis was also carried out manually [12-14]. At the same time, naive maps were often not the only source of information for research: in addition to the maps, informants also completed pre-designed surveys and face-to-face interviews or participated in focus groups $[15,16]$. The processing and analysis of the results were carried out in manual mode.

Later, research began to be carried out using computer technology. In particular, in 1984, S. Waterman and D. Gordon used a computer to calculate the degree to which a digitized naive map matched an objective geographic map of Israel based on a set of control points [17]. F. Aram et al. describe a more comprehensive approach to the analysis of naive maps considering a set of landmarks and paths, which were marked on the maps by informants. Objects are analyzed from two aspects: location accuracy and map complexity. Accuracy is determined by the degree of correspondence between the sets of landmarks and paths, marked by informants, with the sets predetermined by experts. The degree of correspondence is determined by the percentage ratio of the number of objects from the expert list found on the naive maps of informants to the length of the expert list. Complexity is measured using the cell percentage method and based on Appleyard's scoreboard. The program proposed by Aram et al. makes it possible to both compare the naive map with the one created by the experts (or with another naive map) and get the reflection of information from all the informant maps to the objective geographical map [18]. However, despite the fact that the analysis of the maps was carried out on a computer, the creation of the maps took place on paper.

Most researchers distinguish three main components in naive maps: lines, points, and zones [18]. Indeed, when creating a two-dimensional map, any object can be represented by one of these three components or their combination. In some cases, informants draw $3 \mathrm{D}$ naive maps. However, such maps are usually analyzed when 
the dimensions of the investigated space are comparable to the heights of objects. Accordingly, when the object of study is a relatively large geographic area, it is possible to neglect information about the heights of objects and use a two-dimensional space. The methods used for processing naive maps depend on the dimensions of space.

When the sample of maps is small enough, then usually the research is carried out at a qualitative level and ad-hoc methods are used. Otherwise, one or more of the basic approaches to map analysis are used. One of these approaches is based on comparing naive maps with a real geographical map. Another approach is to compare the maps of informants with each other to identify some trends. A combination of these approaches is sometimes used [19].

The basic element for comparison in these cases is a set of landmarks on the map. On a global scale, such landmarks can be, for example, settlements, state borders, seas, mountain ranges, etc.

The choice of comparison methods depends on the form of data presentation. When objects are deterministic, it is convenient to use the bidimensional regression method, which makes it possible to compare two two-dimensional objects with each other [20]. A naive map can also act as an object. A. L. Gardony et al. used, in addition to the bidimensional regression method, the method of pairwise comparison of landmarks [21]. At the same time, the initial naive maps are created on paper and, accordingly, are analyzed visually. If the pattern or naive map is fuzzy, it makes sense to use methods of fuzzy classification of objects. One of such methods is the weighted fuzzy pattern matching [22]. Although it is used in its original form to compare fuzzy numbers, it can be applied to compare the corresponding numerical parameters of a naive map and a pattern.

We propose the Creative Map Studio, which provides tools for creating and analyzing naive maps. Maps created in this application are digital, and they can be analyzed without manually marking the landmarks. The objects created in the studio have meta-information not inlined on the map. So, they can be verbally described preserving the clean visualization of the map. In addition, the naive maps created in this application store both the final state of the map and the history of its creation.

The analytics available for the maps drawn in Creative Map Studio relies on SciVi data mining platform [23] and Semograph graph-semantic modeling information system for textual data processing [24]. Both of these software systems were created in our previous research and proved their abilities of an efficient and comprehensive analysis of complex and variable data [9].

SciVi data mining platform (https://scivi.tools) is built on the principles of ontology engineering. Its functioning is completely governed by an ontological knowledge base, in which all the data processing, analysis, and visualization capabilities are described. Thereby, SciVi is a very flexible toolset for solving analytics tasks in different application domains because it can be extended with the new functionality by modifying its knowledge base only [25]. The data processing pipelines in SciVi are declared in a high-level graphical user interface leveraging intuitive visual programming language based on data flow diagrams [25]. SciVi usage requires no specific IT skills, which makes this platform available for a wide range of experts in different scientific fields.

The Semograph graph-semantic modeling information system (https://semograph.com) is built on the principle of data representation in the form of a graph, which vertices reflect the semantic cores of concepts, and edges characterize the semantic connections between concepts. The essence of its functioning is that the data loaded for analysis are divided into some semantic parts (such as book chap- 
ters, individual objects within the dataset, etc.). These parts are called "contexts". Within each context, "semantic fields" and "semantic components" are distinguished. Semantic fields are lexemes or combinations of lexemes. Semantic components are the entities associated with the fields, which reflect the presence of certain semantics in the corresponding fields. Semograph has a high-level graphical user interface with which the user can both manually and automatically work with text data, studying their semantic properties. Analyzing textual data based on semantic fields and semantic components reveals its semantic structure that is an important part of linguistic research $[26,27]$.

\section{Creative Maps Studio Architecture}

Creative Maps Studio is a client-server web application designed to work with naive maps representing spatial mental maps. The main concepts of Creative Maps Studio architecture are presented in a form of ontology in Fig. 1.

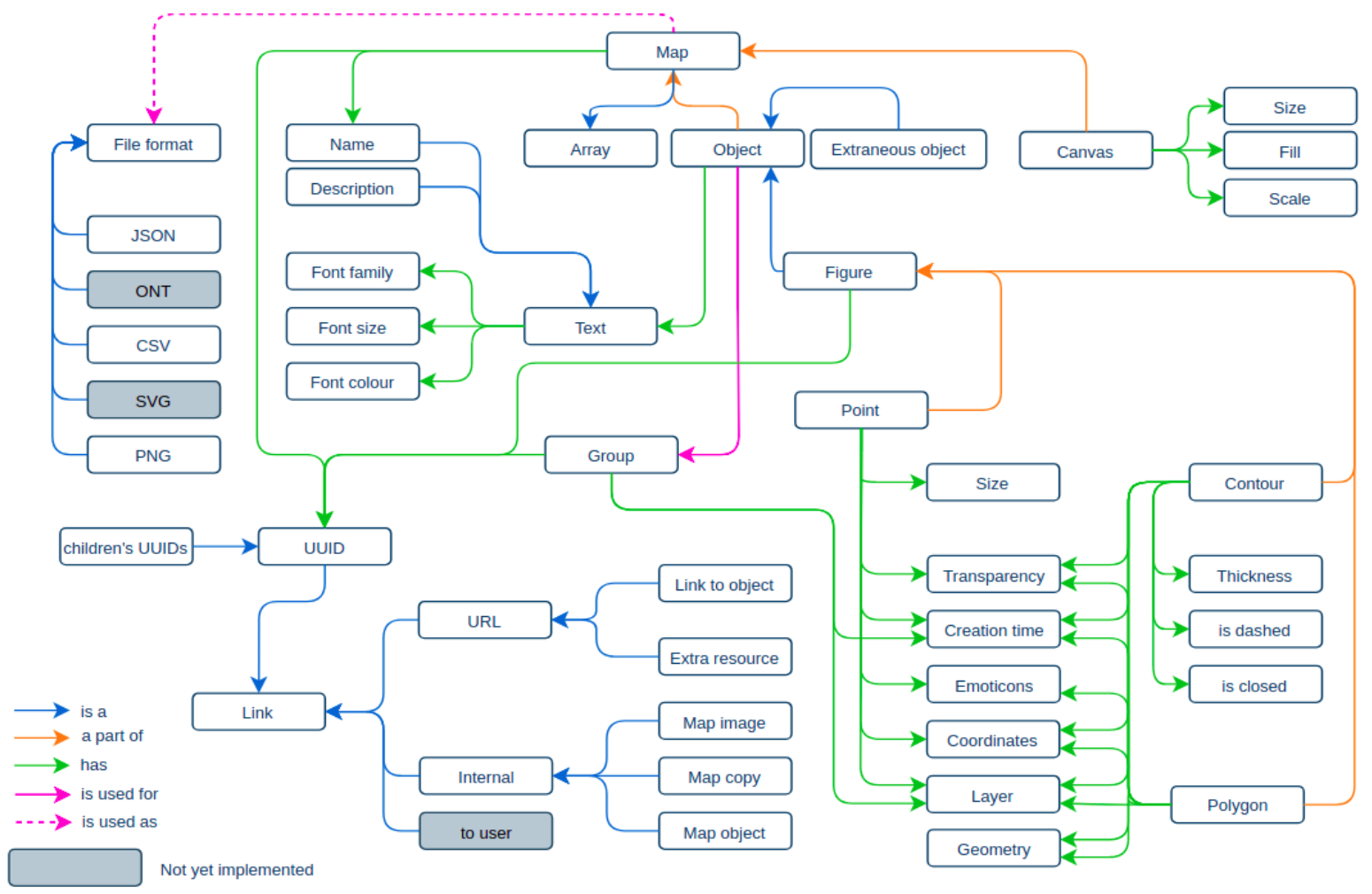

Fig. 1. Ontology of Creative Maps Studio

With the help of Creative Maps Studio, the informant can digitally draw a geomental representation of a certain space, which reflects a mental map of his/her spatial perception. A mental map is a complex object that represents a certain geoconcept and contains a set of graphical objects with their verbal descriptions, which characterize the informant's subjective attitude to space. The physical representation of a mental map is a naive map. On the one hand, a naive map represents geographical objects as some spatial entities, which can be studied using various analytical methods, and as a result, give some semantic interpretation of the location and parameters of objects. On the other hand, geographical objects contain a text layer that makes it possible to highlight the informant's attitude to objects and allows researchers to match the drawn objects with the real ones and identify the depicted space, 
that is, to determine the map's belonging to some geo-concept (or a combination of geo-concepts).

In addition, the informant can provide information about himself. Such information makes it possible to investigate differences in the content or presentation form of mental maps based on some characteristics of the informants, such as gender, region of residence, etc.

The Creative Maps Studio is available at https://creativemaps.studio. Moving on to the technical aspects of its functioning, we note that maps are internally stored in JSON format and can be exported to CSV tabular format and raster PNG format. While Creative Maps Studio stores the map objects with the full history of creation and manipulations, when exporting to CSV, only the final states of the objects are dumped. If some specific data conversion is required, additional conversion modules are used. The modules are written in Python and executed on the server side as processing plugins. For example, plugins are used to normalize parameters among a sample of maps.

The server side of Creative Maps Studio consists of the following components: main module (written in Python 3 using FastAPI), storage module (based on PostgreSQL), and caching module (based on Redis). The client side is implemented in JavaScript using React, Redux, and Gatsby using HTML5 templates and CSS3 styling. The architecture diagram is shown in Fig. 2.

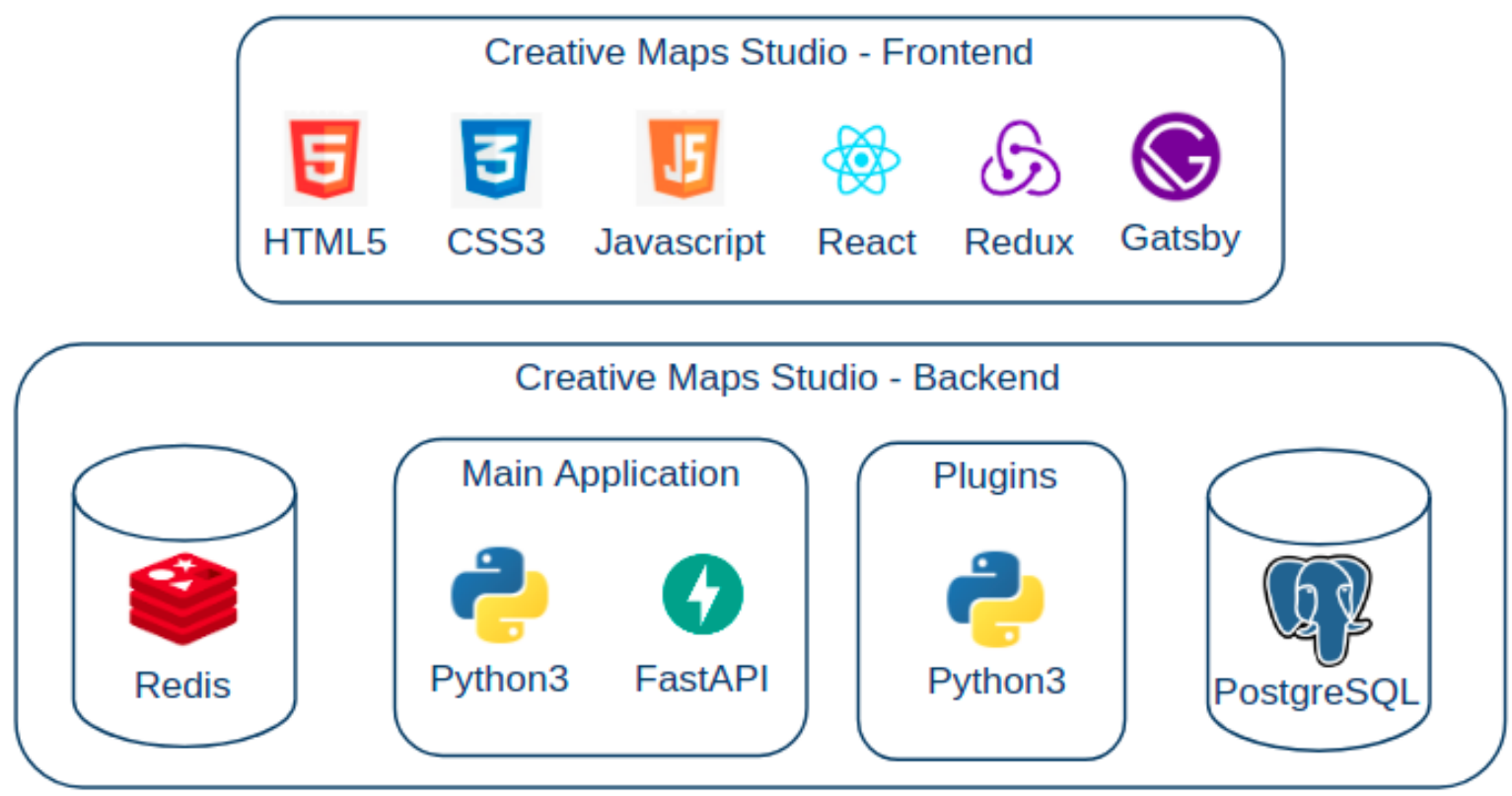

Fig. 2. Creative Maps Studio architecture diagram

The server provides the following functions:

1. Authorizing and registering the users directly in the application and $\log$ in with their credentials, or log in to the application via their profile on the following social networks: 'ВКонтакте' (Vkontakte), Facebook and 'Одноклассники' (Odnoklassniki).

2. Storing the maps drawn by the user and providing access to them (but users can also draw maps anonymously and save these maps at their computers).

3. Providing access to copies of maps drawn by other users by direct links.

4. Performing requests to third-party resources: 
a. Database of settlements in Russia.

b. Social networks, through which users can log in to the studio and in which they can share maps they have drawn.

The client provides the tools for creating and editing maps. It is possible to arrange the following types of objects on the map: point objects such as cities, cultural monuments, and industrial objects; large-scale objects such as bodies of water, deserts, and swamps; extended objects such as state borders, roads, and rivers. Objects can be given individual visual characteristics, as well as a verbal name and description. Additionally, it is possible to indicate attitude to the objects using emoticons. Drawn maps contain not only the final state of all objects but also the history of all manipulations with objects performed during drawing. The manipulations' history allows researchers to analyze the process of creating a map. In addition, in the studio client, the user can receive training on working in the studio or refer to the help on using it.

\section{Digital Drawing of Naive Maps: Pros and Cons}

Since naive maps are presented in graphical form, they can be created both on a physical medium, for example, on paper, and in a digital form. Let us compare digital and paper-drawn naive maps.

Firstly, let us look at the advantages of digital naive maps over paper-drawn ones:

1. Objects on digital maps can be interpreted with higher accuracy than on paper-drawn ones: digital maps have limited variation in their parameters, so they can be better classified and have no issues with recognition of handwriting.

2. Digital maps can be analyzed automatically (no conversion to the digital format is needed). This is especially important for large map arrays.

3. Digital maps contain easily retrievable text information (names, descriptions, emoticon-based expressions about objects) without compromising the visualization of map objects. This is difficult for paper-drawn maps, where all the text must always be visible on the map.

4. Digital maps are easier to store and back up: they take up a small amount of space on the digital storage and can be copied fast and losslessly, while paper-drawn maps require large physical storage with special conditions like temperature and humidity, as well as require much more time and effort for backup.

5. Digital maps are created online thus remotely, allowing researchers to involve geographically distant informants.

6. Digital maps make it easier to attract informants to participate in the study since it is more convenient for the informants to draw from home at any time (while the study is being carried out) than to come to the laboratory at a designated time or to transfer the physical map medium in any physical way, or digitize it and send it per e-mail.

7. Digital maps make it possible to overlay objects on top of each other without distorting or losing information about any of them, which allows researchers to get more information from a digital map than from a paper-drawn one.

8. Digital maps are able to save the history of the informant's actions in the drawing process, which is nearly impossible to achieve when using paper-drawn maps.

However, despite all the advantages of digital maps over paper-drawn ones, digital maps also have disadvantages: 
1. Working with paper is more familiar to most of the informants since most users are better at using the tools for traditional drawing (for example, a pencil) than a mouse/touchpad. As a consequence, digital maps usually have less detail and complexity of the contours of objects.

2. Working with digital tools to create digital maps requires training that allows the informants to get used to the interface. If the training time is insufficient or the interface is too complex, there is a high risk of receiving less information from the maps, since informants will not know how to create objects they want.

3. Drawing a digital map is limited by the set of drawing tools. Informants may want to display an object that has not been accounted for by the researchers. In this case, the object may not be reflected on the map, reflected incorrectly (using a tool that does not imply such interpretation), or displayed too abstractly.

Based on the above comparison, we conclude that in most cases digital maps are preferable for building naive maps. Creative Maps Studio allows to benefit from all the above advantages of digital maps, thus it is an adequate toolset for conducting corresponding experiments. As for the disadvantages, the first of them is currently assumed to be impossible to overcome in the short term. The second one can be tackled by the detailed help for the studio and the built-in learning mechanism. The influence of the third one is supposed to be gradually reduced using feedback from informants and adding missing object types.

We handled paper-drawn maps in our previous research in the Digital Humanities field and we can state that maps in this form are difficult to collect and analyze, in particular, it is difficult to extract and process the related textual information [2]. Therefore we believe that the use of digital maps can improve the research process and deepen the data analysis.

\section{Visual Analytics of Mental Maps} maps:

We propose the following ways to analyze the mental maps represented as naive

1. Viewing the map and the process of its creation in Creative Maps Studio (an example is shown in Fig. 3 and available at https://creativemaps.studio/share?share token=eyJ1dWlkIjoiZjA5MjgyMjYtNGNIY iooMTVjLWI4M2UtY2ExNjYyMTcxZGYyIiwidmVyc2lvbiI6MSwidGlobGUiOiLQnd C-oLLQsNGPINC6oLDRgNGCoLAifQ.sapGTjAquMSAlng5XbQW-1DODjU).

2. Visual analytics of maps' set in the SciVi data mining platform.

3. Visual analytics of the maps' text layers in the Semograph information system.

4. Fuzzy classification of naive maps using SciVi.

Further, we will take a closer look at the analytical approaches used by SciVi and Semograph systems. 


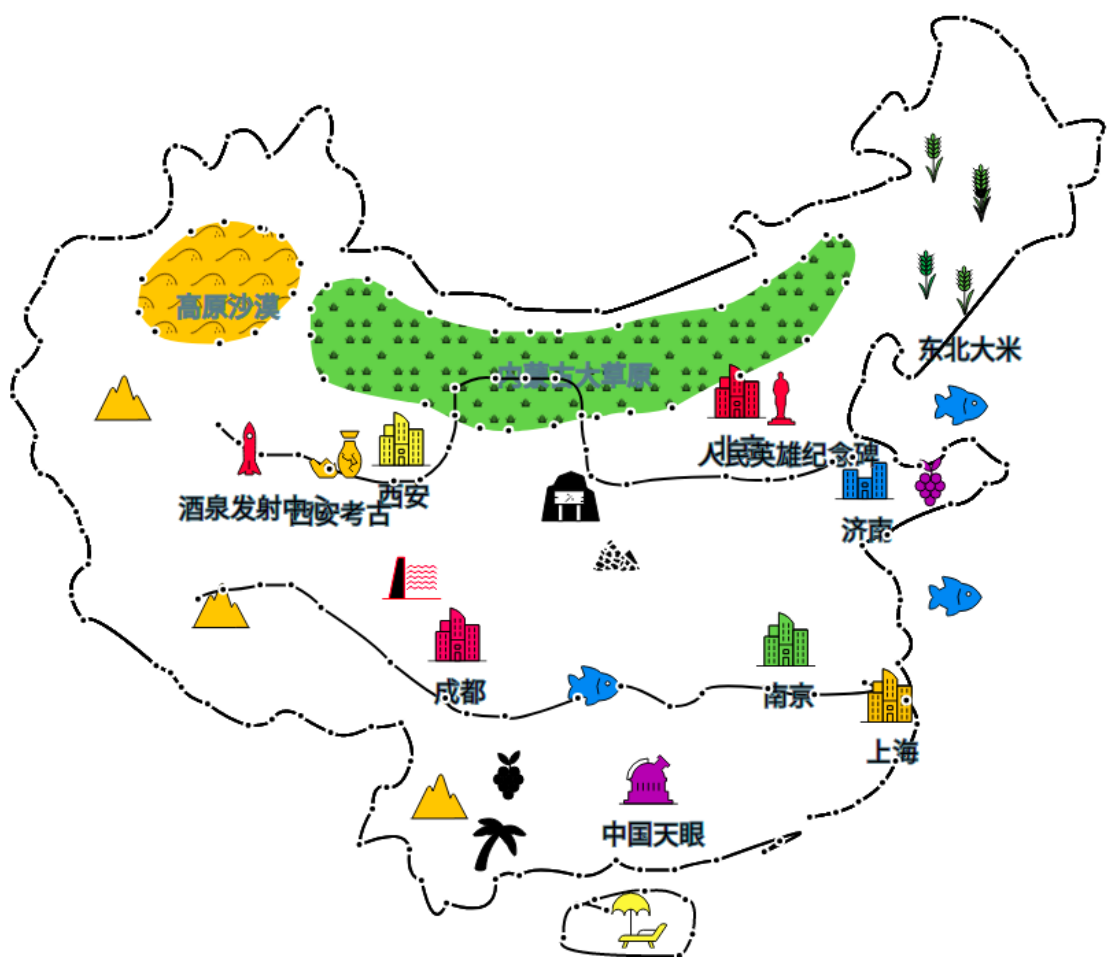

Fig. 3. An Example of a naive map of China drawn in Creative Maps Studio

\subsection{Visual Analytics of Naive Maps in SciVi}

One way to analyze naive maps is to handle them in SciVi. To do this, the parameters needed for analysis are selected from the source maps drawn by the informants, exported to CSV, and loaded into SciVi, where the subsequent analytics pipeline is visually declared using data flow diagrams [25].

As an example of this approach, we studied a set of China maps drawn by 100 students of 2 Chinese universities. A table of the different use colors for some Chinese cities was considered. In this case, the color and name of the city were selected as target parameters to build an aggregated table of maps. However, it is possible to analyze any combination of parameters of objects on the map.

Accordingly, on the SciVi side, a file with information from the maps is fed to the input, and the nodes responsible for the rendering method are selected. In our case, a circular graph is used. Fig. 4 shows the SciVi data flow diagram that allows the input CSV file to be visualized as a circular graph.

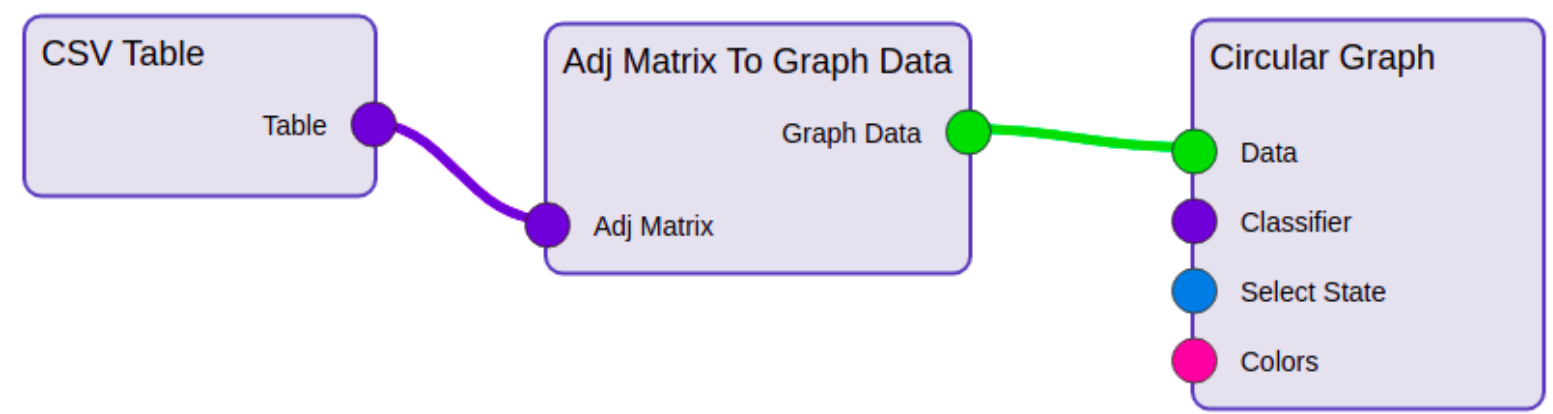

Fig. 4. SciVi data flow diagram 
Executing this data flow diagram, SciVi displays a circular graph that shows which colors are used for each of the cities that the informants drew on their maps. Fig. 5 shows an example of such a circular graph. The color diversity is clearly seen in the graph, while Beijing is most often drawn red.

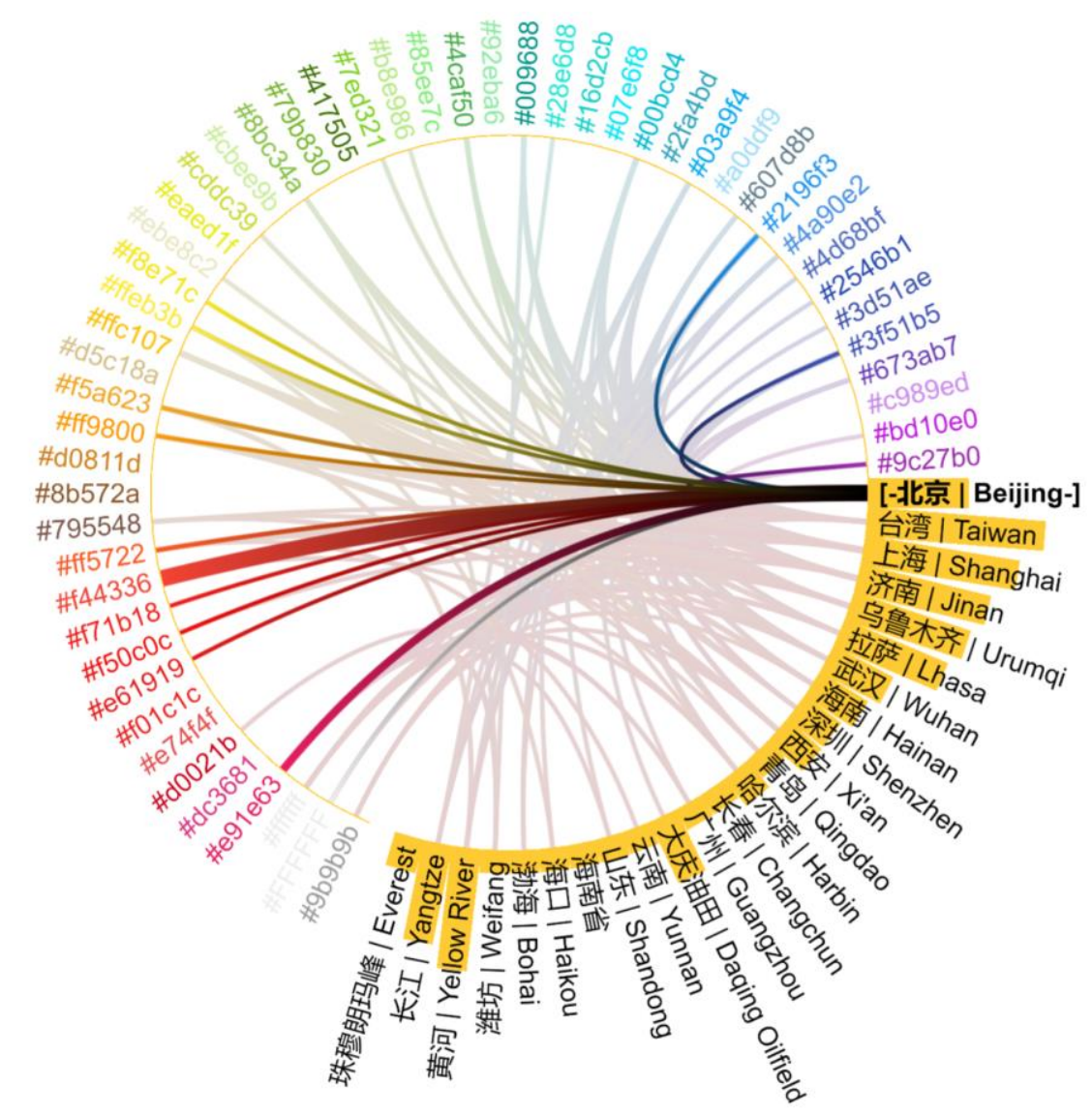

Fig. 5. SciVi Circular graph of using different colors for some Chinese cities

\subsection{Visual Analytics of Naive Maps in Semograph}

Semograph analyzes the textual components of maps, therefore, the main target in such a study is in the analysis of names, descriptions, and emoticons related to the map objects. Accordingly, after loading, Semograph prepares data as follows. The first step is splitting the file into contexts (each context is one map from the file). Within each context, semantic fields (lexemes or their combinations) and semantic components are distinguished. Semantic components are associated with fields and reflect the presence of certain semantics in them. The preparation of the textual data of maps can be carried out by an expert in manual mode, using machine learning methods, or using a combination of these approaches.

After the Semograph has made the primary processing of information from the file, a semantic map is drawn up, which quantitatively reflects the connections between semantic fields. The semantic map can then be visualized by means of the SciVi system in the form of a semantic graph, which makes it possible to visually evaluate the semantic relationships of the semantic fields. Fig. 6 shows an example of a graph representing the semantic structure of the Beijing geo-concept. This geoconcept was reconstructed according to the text layers of the set of China maps (the map collection is the same as described in Section 6.1). In the graph shown in Fig. 6, nodes depict the most frequent terms characterizing the Beijing geo-concept and 
edges depict the simultaneous appearance of corresponding terms within a text layer of the same map. This kind of graph-based visualization allows experts to reveal interesting regularities in the semantic structure across different geo-concepts. For example, it was found out that the Chinese students indicate all the country locations rather positively, expressing quite nothing negative. However, such geo-concepts as Beijing and Shanghai have a much reacher semantic structure than Taiwan that is represented poorly.

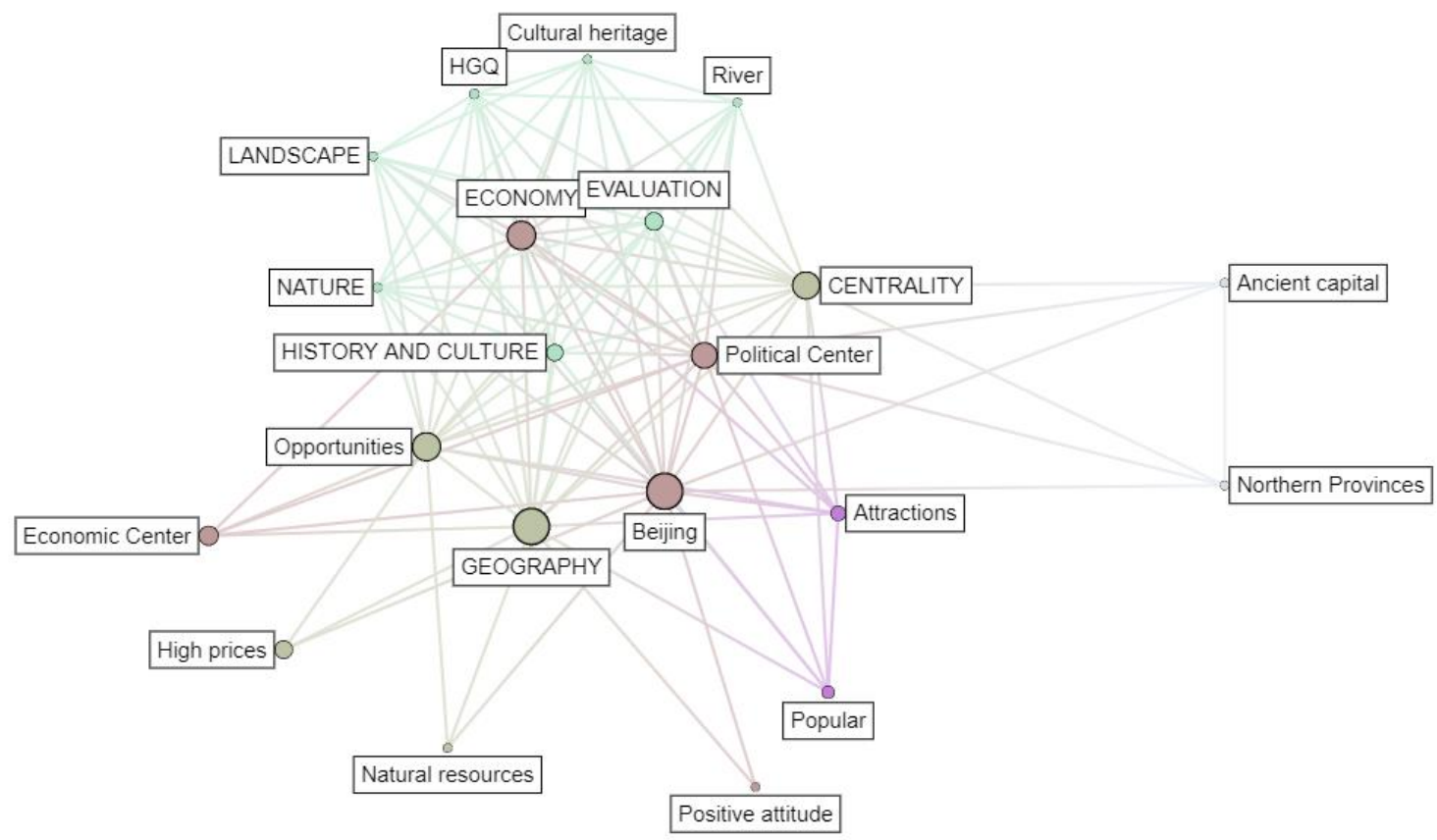

Fig. 6. SciVi semantic graph of Beijing geo-concept

\subsection{Fuzzy Classification of Naive Maps in Scivi}

The fuzzy classification described in this paper is based on the algorithm of weighted fuzzy pattern matching presented in [22]. The described algorithm was implemented as a SciVi plugin written in JavaScript.

Since the algorithm uses a system of weights for the parameters, a weight vector is also compiled before starting. The values of each of the weights are determined based on the importance of the corresponding parameters, as well as taking into account the spread of their values in the set of maps (the smaller the spread, the less weight should be assigned to the parameter in order to avoid anomalous influence on the classification results).

The preprocessed maps are loaded into SciVi in two parts. The first part contains maps, drawn by informants with known regions of residence. These maps represent patterns that characterize different regions. A region pattern is a set of such objects that are found on the maps of the informants from this region. In this case, the parameters of the pattern objects are fuzzy values. The membership function of a certain parameter of a certain object is built according to the values of this parameter for this object, found on different maps of a given region (for details, see [28]). In terms of machine learning, this set of maps is treated as a training sample. Along with the training sample, a vector of parameter weights is provided. The second part of the maps is a set of maps, drawn by informants with unknown residence regions (the test sample of maps). This set of maps is classified. 
Next, SciVi performs pattern building and then sequentially compares each test map with each pattern [28]. According to the results of the classification, a dictionary of the correspondence degrees to each of the patterns is found out for each test map. The highest degree of pattern matching among the dictionary elements is taken as a result of classification for a given test map.

After all the maps have been classified, SciVi visualizes the classification results as a histogram (see Fig. 7). Each bar represents the similarity of the corresponding map to the corresponding region. This fuzzy classification algorithm allows testing the similarity of the given mental map to the maps, which are typical for a certain region. In Fig. 7, the results of fuzzy classification for Perm and Orenburg maps are presented.

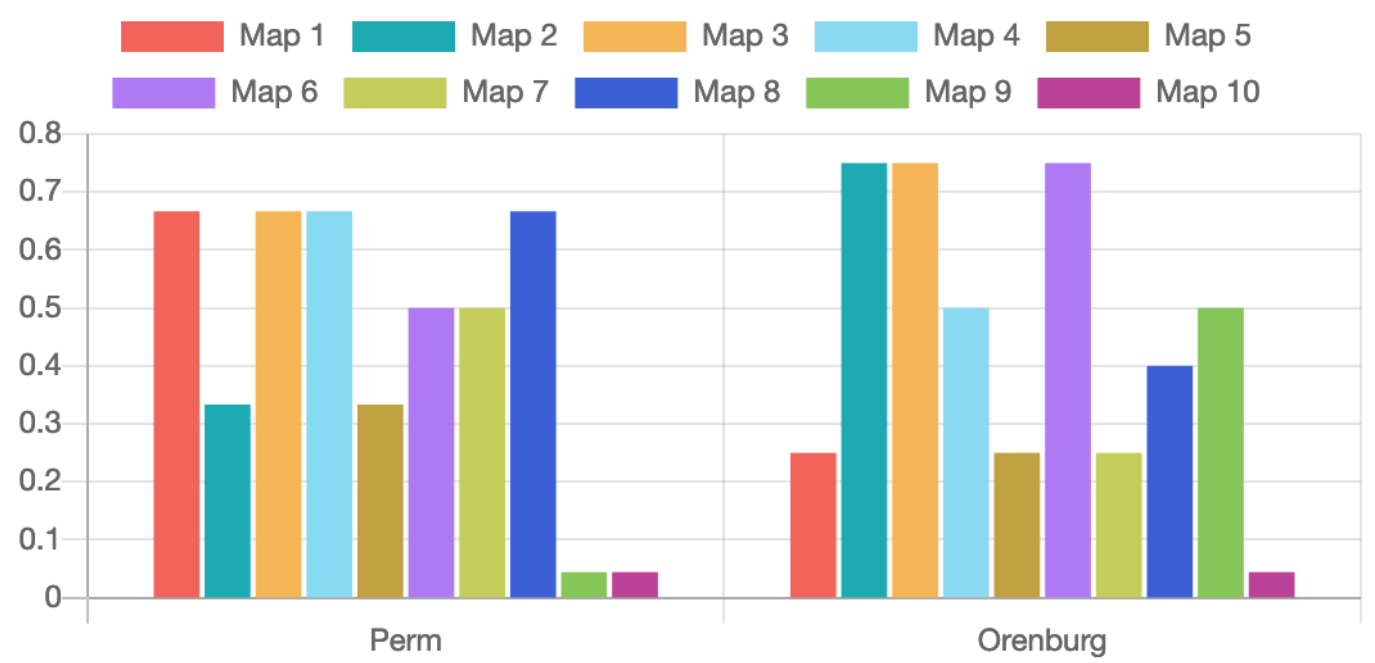

Fig. 7. Visualization of maps' fuzzy classification results in SciVi

\section{Conclusion}

In this paper, we describe the Creative Maps Studio application for digital mental mapping and the ways it can be used to work with digital mental maps presented in a form of naive geographical maps. Digital maps have a number of advantages over the traditional paper-drawn ones, enabling researchers to benefit from the modern data mining methods by analyzing different graphical and semantic properties of these maps. Such an analysis is an essential part of the Digital Humanities research that targets public mood in a certain region, features of human perception of the surrounding regional space, etc.

We propose several approaches to visual analysis of mental maps, involving Creative Maps Studio vector graphics editor, SciVi data mining platform, and Semograph information system. This software tools' combination leverages a comprehensive study of the mental maps' features, which can be extracted from the naive maps' representations.

Currently, we carried out several experiments based on mental maps' analysis. The most significant ones, for now, are the analysis of China maps drawn by the Chinese students, and the fuzzy classification of Russian maps drawn by informants from Perm and Orenburg. The experiments conducted shown the efficiency of the analytical pipelines proposed. We can state, that digital naive maps composed in the Creative Maps Studio appear to be adequate visualization of subjective geospatial representations of a person and social groups, and the described software combina- 
tion is a viable toolkit for extracting information from the constructed mental map models.

In the future, we plan to extend the number of operators to analyze mental maps in SciVi and Semograph, as well as to explore the ways of analyzing the history of maps' objects manipulations within Creative Maps Studio. Also, we plan to expand the set of objects available for drawing the maps and implement the mechanism allowing researchers to create custom map elements for particular needs.

\section{Acknowledgements}

This work was supported by Russian Science Foundation (grant number 20-18oo336).

\section{References}

1. Henrikson, A.K. The Geographical "Mental Maps" of American Foreign Policy Makers. International Political Science Review // Polytics and Geography. - 1980. Vol. 1, No. 4. - PP. 495-530. DOI: 10.1177/019251218000100405.

2. Zelianskaia, N.L., Belousov, K.I., Galinskaia, T.N., Ichkineeva, D.A. Naive Geography: Geoconceptology and Topology of Geomental Maps // Heliyon. - 2020. Vol. 6, Iss. 12. DOI: 10.1016/j.heliyon.2020.e05644.

3. Self, C.M., Gopal, S., Golledge, R.G., Fenstermaker, S. Gender-Related Differences in Spatial Abilities // Progress in Human Geography. - 1992. - Vol. 16, No. 3. - PP. 315-342. DOI: 10.1177/030913259201600301.

4. Kaplan, D.H., Herb, G.H. How Geography Shapes National Identities // National Identities. - 2011. - Vol. 13, No. 4. - PP. 349-360. DOI: 10.1080/14608944.2011.629424.

5. Taylor, S.A. Place Identification and Positive Realities of Aging // Journal of Cross-Cultural Gerontology. - 2001. - No. 16. - PP. 5-20. DOI: 10.1023/a:1010673000367.

6. Saarinen, T.F., MacCabe, C.L. World Patterns of Geographic Literacy Based on Sketch Map Quality // The Professional Geographer. - 1995. - Vol. 47, Iss. 2. PP. 196-204. DOI: 10.1111/j.0033-0124.1995.00196.x.

7. Didelon, C., de Ruffray, S., Boquet, M., Lambert, N. A World of Interstices: A Fuzzy Logic Approach to the Analysis of Interpretative Maps // The Cartographic Journal. - 2011. - Vol. 48. - PP. 100-107. DOI: 10.1179/1743277411Y.0000000009.

8. Blades, M. The Reliability of Data Collected from Sketch Maps // Journal of Environmental Psychology. - 1990. - Vol. 10, Iss. 4. - PP. 327-339. DOI: 10.1016/So272-4944(05)80032-5.

9. Ryabinin, K.V., Belousov, K.I., Chuprina, S.I., Shchebetenko, S.A., Permyakov, S.S. Visual Analytics Tools for Systematic Exploration of MultiParameter Data of Social Web-Based Service Users // Scientific Visualization. 2018. - Vol. 10, No. 4. - PP. 82-99. DOI: 10.26583/sv.10.4.07.

10. Lynch, K. The Image of City. $-1960 .-197 \mathrm{p}$.

11. Gould, P.R. On Mental Maps. - 1966. - 106 p.

12. Milgram, S., Jodelet, D. Psychological Maps of Paris // Environmental psychology: People and their physical settings. - 1976. - PP. 88-113.

13. Herman, J.F. Children's Cognitive Maps of Large-Scale Spaces: Effects of Exploration, Direction, and Repeated Experience. - 1980. - Vol. 29, No. 1. - PP. 126143. DOI: 10.1016/0022-0965(80)90096-X. 
14. Evans, G.W., Marrero, D.G., Butler, P.A. Environmental Learning and Cognitive Mapping // Environment and Behavior. - 1981. - Vol. 13, Iss. 1. - PP. 83-104. DOI: 10.1177/0013916581131005.

15. Gieseking, J.J. Where We Go from Here: The Spatial Mental Mapping Method and Its Analytic Components // Qualitative Inquiry. - 2013. - Vol. 19, No. 9. - PP. 712-724. DOI: $10.1177 / 1077800413500926$.

16. White, R.J., Green, A.E. The Use of Mental Maps in Youth Research: Some Evidence from Research Exploring Young People's Awareness of and Attachment to Place // Innovations in Youth Research. - 2012. - PP. 58-76. DOI:10.1057/9780230355880_4.

17. Waterman, S., Gordon, D. A Quantitative-Comparative Approach to Analysis of Distortion in Mental Maps // Professional Geographer. - 1984. - Vol. 36, No. 3. PP. 326-337. DOI: 10.1111/j.0033-0124.1984.00326.x.

18. Aram, F., Solgi, E., García, E.H., Mohammadzadeh, D., Mosavi, A., Shamshirband, S. Design and Validation of a Computational Program for Analysing Mental Maps: Aram Mental Map Analyzer // Sustainability. - 2019. - Vol. 11, No. 14. DOI: $10.3390 /$ su11143790.

19. Appleyard, D. Styles and Methods of Structuring a City // Environment and Behavior. - 1970. - Vol. 2, No. 1. PP. 100-117. DOI:10.1177/001391657000200106.

20. Tobler, W.R. Bidimensional Regression // Geographical Analysis. - 1994. Vol. 26, No. 3. PP. 187-212. DOI: 10.1111/j.1538-4632.1994.tbo0320.x.

21. Gardony, A.L., Taylor, H.A., Brunyé, T.T. Gardony Map Drawing Analyzer: Software for Quantitative Analysis of Sketch Maps // Behavioral Research Methods. - 2015. - Vol. 48, No. 1. - PP. 151-177. DOI: 10.3758/s13428-014-0556-x.

22. Dubois, D., Prade, H., Testemale, C. Weighted Fuzzy Pattern Matching // Fuzzy Sets and Systems. - 1988. - Vol. 28, No. 3. - PP. 313-331. DOI:10.1016/01650114(88)90038-3.

23. Ryabinin, K., Chuprina, S. Development of Ontology-Based Multiplatform Adaptive Scientific Visualization System // Journal of Computational Science. 2015. - Vol. 10. - PP. 370-381. DOI: 10.1016/j.jocs.2015.03.003.

24. Belousov, K., Erofeeva, E., Leshchenko, Y., Baranov, D. "Semograph" Information System as a Framework for Network-Based Science and Education // Smart Innovation, Systems and Technologies. Smart Education and e-Learning. - 2017. PP. 263-272. DOI: 10.1007/978-3-319-59451-4_26.

25. Ryabinin, K.V., Belousov, K.I., Chuprina, S.I. Novel Circular Graph Capabilities for Comprehensive Visual Analytics of Interconnected Data in Digital Humanities // Scientific Visualization. - 2020. - Vol. 12, No. 4. - PP. 56-70. DOI: 10.26583/sv.12.4.06.

26. Baranov, D., Belousov, K., Erofeeva, E., Leshchenko, Y. Semograph Information System as a Platform for Network-Based Linguistic Research: A Case Study of Verbal Behavior of Social Network Users // Springer. - 2019. - Vol. 144. - PP. 313324. DOI: 10.1007/978-981-13-8260-4_29.

27. Zelyanskaya, N., Galinskaia, T., Belousov, K. Vladimir Putin's Image of the World: 'Apophatic' Thinking as a Political Principle // International Multidisciplinary Scientific Conference on Social Sciences and Arts SGEM2014. - 2014. - B. 2, Vol. 1. - PP. 483-490. DOI: 10.5593/sgemsocial2014/B21/S4.064.

28. Ryabinin, K.V., Belousov, K.I., Chumakov, R.V. Ontology-Driven Data Mining Platform for Fuzzy Classification of Mental Maps // Frontiers in Artificial Intelligence and Applications. - Accepted for publication in 2021. 